\title{
A GLOBAL PINCHING THEOREM OF MINIMAL HYPERSURFACES IN THE SPHERE
}

\author{
SHEN CHUN-LI
}

(Communicated by David G. Ebin)

\begin{abstract}
Let $M^{n} \subset S^{n+1}(1)$ be a compact embedded minimal hypersurface in the sphere $(n \geq 3)$, and $\sigma$ the square of the length of the second fundamental form of $M^{n}$. Suppose $M^{n}$ has nonnegative Ricci curvature. Then there is a constant $A(n)$, depending only on $n$, such that if $\|\sigma\|_{n / 2}<A(n)$, then $M^{n}$ must be totally geodesic. Here $\|\sigma\|_{K}=\left(\int_{M} \sigma^{K}\right)^{1 / K}$. It is related to the results of J. Simons [6] and S. T. Yau [9] about the minimal hypersurfaces in the sphere. For the case $n=2$, we also have a similar discussion.
\end{abstract}

As it is known to all, J. Simons [6] has proved that if $\sigma$, the square of the length of the second fundamental form of compact minimal submanifold $M^{n}$ in the sphere $S^{n+1}(1)$, satisfies $0 \leq \sigma<n$, then $M^{n}$ must be totally geodesic. S. T. Yau [9] also investigated the similar problem, and has proved that if the compact minimal submanifold $M^{n}$ in sphere $S^{n+1}(1)$ has positive sectional curvature, then $M^{n}$ must be totally geodesic. In this paper, we try to improve these results. In J. Simons' theorem, the condition $0 \leq \sigma<n$ must be pointwise satisfied. We try to replace this pointwise assumption by an integral expression of $\sigma$, and replace the condition of positive sectional curvature of $M$ in Yau's theorem by the nonnegative Ricci curvature of $M^{n}$. Therefore we obtain the following global pinching theorems of minimal hypersurfaces in the sphere.

Theorem 1. Let $M^{n}(n \geq 3)$ be a compact embedded minimal hypersurface of sphere $S^{n+1}(1)$. Suppose $M^{n}$ has nonnegative Ricci curvature. Let $\sigma$ be the square of the length of the second fundamental form of $M$. Then there is a constant $A(n)$ depending only on $n$ (see (27) below), such that if $\|\sigma\|_{n / 2}<$ $A(n)$, then $\sigma=0$, i.e., $M^{n}$ must be totally geodesic. Here

$$
\|\sigma\|_{K}=\left(\int_{M} \sigma^{K}\right)^{1 / K}
$$

Theorem 2. Let $M^{2}$ be a compact embedded minimal surface with genus $g$ in sphere $S^{3}(1)$. Suppose $M^{2}$ has nonnegative Gauss curvature. Let $\sigma$ be the square of the length of the second fundamental form of $M$. Then there is a

Received by the editors October 1, 1987 and, in revised form, December 26, 1987.

1980 Mathematics Subject Classification (1985 Revision). Primary 53C42; Secondary 53C40. 
constant $A=1 /(6912 \sqrt{2 \pi(g+1)})$, such that if $\|\sigma\|_{2}<A$, we have $\sigma=0$, i.e., $M^{2}$ must be totally geodesic (this time, $g$ must be zero).

Proof of Theorems 1 and 2.

1. We use the method of moving frame. Let $\left\{e_{1}, \ldots, e_{n}\right\}$ be an orthonormal base of $M^{n} \subset S^{n+1}(1)$, and let $h_{i j}(i, j=1, \ldots, n)$ be the second fundamental form of $M^{n}, \sigma$ the square of the length of $h_{i j}$, i.e.,

$$
\sigma=\sum_{i, j=1}^{n} h_{i j}^{2}
$$

We denote the covariant derivatives of $h_{i j}$ by $h_{i j k}, h_{i j k l}, \ldots$, etc. According to J. Simons [6], we can compute the Laplacian of $\sigma$ and obtain that

$$
\frac{1}{2} \Delta \sigma=\sum_{i, j, k} h_{i j k}^{2}+n \sigma-\sigma^{2} .
$$

2. Using the Schwarz inequality, we have

$$
\sum\left(h_{i j k}\right)^{2} \geq|\operatorname{grad} \sqrt{\sigma}|^{2} .
$$

Hence

$$
\frac{1}{2} \Delta \sigma \geq|\operatorname{grad} \sqrt{\sigma}|^{2}+n \sigma-\sigma^{2} .
$$

Integrating both sides of (3), we obtain that

$$
0 \geq|\operatorname{grad} \sqrt{\sigma}|^{2}+n \int \sigma-\int \sigma^{2} .
$$

3. Let us state the following Sobolev inequality obtained by P. Li [5]:

For every $f \in H_{1,2}\left(M^{n}\right), n=\operatorname{dim} M>2$, we have

$$
\begin{aligned}
\int|\operatorname{grad} f|^{2} \geq\left(\frac{n-2}{2(n-1)}\right)^{2} C_{0}^{2 / n}\left[2^{-(n+2) / n}\|f\|_{2 n /(n-2)}^{2}\right. & \\
& \left.-(\operatorname{vol} M)^{-2 / n} \cdot 2^{E(n)}\|f\|_{2}^{2}\right],
\end{aligned}
$$

where

$$
E(n)=\left\{\begin{array}{l}
1, \quad n=3, \\
(n-4)(n-2) / 2, \quad n>3,
\end{array}\right.
$$

and the best Sobolev constant $C_{0}$ satisfies the following inequality:

$$
C_{1} \leq C_{0} \leq 2 C_{1} \text {. }
$$

In (6), the isoperimetric constant is defined by

$$
C_{1}=\inf _{S} \frac{(\operatorname{Area}(S))^{n}}{\left(\min \left(\operatorname{vol} M_{1}, \operatorname{vol} M_{2}\right)\right)^{n-1}},
$$

where $S$ takes all hypersurfaces of $M, S$ divides $M$ into two parts $M_{1}, M_{2}$, and $\operatorname{Area}(S)$ is the $(n-1)$-dimensional volume of $S$. 
From (5), (6), we have

$$
\begin{aligned}
\int|\operatorname{grad} f|^{2} \geq & \left(\frac{n-2}{2(n-1)}\right)^{2} C_{1}^{2 / n} \cdot 2^{-(n+2) / n}\|f\|_{2 n /(n-2)}^{2} \\
& -\left(\frac{n-2}{2(n-1)}\right)^{2}\left(2 C_{1}\right)^{2 / n}(\operatorname{vol} M)^{-2 / n} \cdot 2^{E(n)} \cdot\|f\|_{2}^{2} .
\end{aligned}
$$

Let

$$
\begin{gathered}
k_{1}=\left(\frac{n-2}{2(n-1)}\right)^{2} C_{1}^{2 / n} \cdot 2^{-(n+2) / n}, \\
k_{2}=\left(\frac{n-2}{2(n-1)}\right)^{2} \cdot\left(2 C_{1}\right)^{2 / n}(\operatorname{vol} M)^{-2 / n} \cdot 2^{E(n)},
\end{gathered}
$$

hence

$$
\int|\operatorname{grad} f|^{2} \geq k_{1}\|f\|_{2 n /(n-2)}^{2}-k_{2}\|f\|_{2}^{2} .
$$

4. From (4), (9), we obtain that

$$
\begin{aligned}
0 & \geq k_{1}\|\sqrt{\sigma}\|_{2 n /(n-2)}^{2}-k_{2}\|\sqrt{\sigma}\|_{2}^{2}+n\|\sigma\|_{1}-\|\sigma\|_{2}^{2} \\
& =k_{1}\|\sigma\|_{n /(n-2)}-k_{2}\|\sigma\|_{1}+n\|\sigma\|_{1}-\|\sigma\|_{2}^{2} \\
& \geq k_{1}\|\sigma\|_{n /(n-2)}+\left(n-k_{2}\right)\|\sigma\|_{1}-\|\sigma\|_{n / 2} \cdot\|\sigma\|_{n /(n-2)} \\
& =\left(k_{1}-\|\sigma\|_{n / 2}\right)\|\sigma\|_{n /(n-2)}+\left(n-k_{2}\right)\|\sigma\|_{1} .
\end{aligned}
$$

5. If we suppose

$$
\|\sigma\|_{n / 2}<\min \left(k_{1}, n \cdot k_{1} / k_{2}\right),
$$

then if $\sigma \neq 0$, by (4), we have

$$
\begin{aligned}
0 & \geq n\|\sigma\|_{1}-\|\sigma\|_{n / 2} \cdot\|\sigma\|_{n /(n-2)} \\
& >n\|\sigma\|_{1}-n \cdot k_{1} / k_{2}\|\sigma\|_{n /(n-2)} .
\end{aligned}
$$

Hence

$$
\|\sigma\|_{n /(n-2)}>\frac{k_{2}}{k_{1}}\|\sigma\|_{1} .
$$

Then by (10), we obtain that

$$
\begin{aligned}
0 & >\left(k_{1}-\|\sigma\|_{n / 2}\right) \frac{k_{2}}{k_{1}}\|\sigma\|_{1}+\left(n-k_{2}\right)\|\sigma\|_{1} \\
& =\left(-\|\sigma\|_{n / 2} \cdot \frac{k_{2}}{k_{1}}+n\right)\|\sigma\|_{1} \\
& >0,
\end{aligned}
$$

so we have a contradiction. Therefore $\sigma=0$, i.e., $M^{n}$ is totally geodesic.

6. When $\operatorname{dim} M=2$, we can use another Sobolev inequality obtained by P. Li [5]: 
For every $f \in H_{1,2}\left(M^{2}\right)$, we have

$$
\begin{aligned}
|\operatorname{grad} f|^{2} & \geq \frac{C_{0}}{4}\left[(\operatorname{vol} M)^{-1 / 2}\left(\int f^{4}\right)^{1 / 2}-(\operatorname{vol} M)^{-1} \int f^{2}\right] \\
& \geq \frac{C_{1}}{4}(\operatorname{vol} M)^{-1 / 2}\|f\|_{4}^{2}-\frac{C_{1}}{2}(\operatorname{vol} M)^{-1}\|f\|_{2}^{2} .
\end{aligned}
$$

Let

$$
k_{1}=\frac{C_{1}}{4}(\operatorname{vol} M)^{-1 / 2}, \quad k_{2}=\frac{C_{1}}{2}(\operatorname{vol} M)^{-1} .
$$

So by (4), (13), we have

$$
\begin{aligned}
0 & \geq k_{1}\|\sigma\|_{2}-k_{2}\|\sigma\|_{1}+2\|\sigma\|_{1}-\|\sigma\|_{2}^{2} \\
& =\left(k_{1}-\|\sigma\|_{2}\right)\|\sigma\|_{2}+\left(2-k_{2}\right)\|\sigma\|_{1} .
\end{aligned}
$$

If we assume

$$
\|\sigma\|_{2}<\min \left(k_{1}, 2 \cdot k_{1} / k_{2}\right),
$$

then if $\sigma \neq 0$, from (4), we get

$$
0 \geq 2\|\sigma\|_{1}-\|\sigma\|_{2}^{2}>2\|\sigma\|_{1}-2 \frac{k_{1}}{k_{2}}\|\sigma\|_{2} .
$$

Consequently,

$$
\|\sigma\|_{2}>\frac{k_{2}}{k_{1}}\|\sigma\|_{1} .
$$

From (15) and (17), we know

$$
0 \geq\left(k_{1}-\|\sigma\|_{2}\right) \frac{k_{2}}{k_{1}}\|\sigma\|_{1}+\left(2-k_{2}\right)\|\sigma\|_{1}>0,
$$

so we have a contradiction. Therefore $\sigma=0$, i.e., $M^{2}$ is totally geodesic.

7. Summarizing the above, when $n \geq 3$, we take a constant

$$
\begin{aligned}
A(n) & =\min \left(k_{1}, n \frac{k_{1}}{k_{2}}\right) \\
& =\min \left(\left(\frac{n-2}{2(n-1)}\right)^{2} \cdot 2^{-(n+2) / n} \cdot C_{1}^{2 / n}, n \cdot 2^{-((n+4) / n+E(n))}(\operatorname{vol} M)^{2 / n}\right),
\end{aligned}
$$

and when $n=2$, we take a constant

$$
A=\min \left(k_{1}, 2 \cdot \frac{k_{1}}{k_{2}}\right)=\min \left(\frac{C_{1}}{4}(\operatorname{vol} M)^{-1 / 2},(\operatorname{vol} M)^{1 / 2}\right) .
$$

Then, Theorems 1 and 2 can be proved. But it is a pity that the isoperimetric constant $C_{1}$ and volume vol $M$ are all dependent on the geometrical properties of $M$. So we want to find the positive lower bounds of isoperimetric constant and volume of minimal hypersurface $M^{n}$ which satisfies the conditions of this 
theorem. Only this way can we find the constants $A(n)$ and $A$ which are needed in this theorem.

8. At first, we want to find the lower bound of vol $M$. According to D. Hoffman and J. Spruck [4], the volume of any compact minimal hypersurface $M^{n}$ in sphere $S^{n+1}(1)$ satisfies

$$
\operatorname{vol} M \geq \alpha(n)
$$

where $\alpha(n)$ is the volume of $n$-dimensional unit sphere. Then the constant $\alpha(n)$ is the positive lower bound of the volume of $M$.

9. Computation of the positive lower bound of the isoperimetric constant $C_{1}(M)$ of $M$.

From C. B. Croke [3], we know that for any compact Riemannian manifold $M$ with the lower bound $(n-1) K$ of Ricci curvature,

$$
C_{1}(M) \geq \frac{1}{4 \alpha(n-1)(\alpha(n))^{n-1}}\left(\frac{\operatorname{vol} M}{\int_{0}^{d}(\sqrt{-1 / K} \sinh \sqrt{-K} r)^{n-1} d r}\right)^{n+1},
$$

where $d$ is the diameter of $M$, and the parenthesis $(\sqrt{-1 / K} \sinh \sqrt{-K})$ means $r$ for $K=0$, and $\sqrt{1 / K} \sin \sqrt{K}$ for $K>0$.

In this theorem we have assumed that $M$ has nonnegative Ricci curvature, so we can take $K=0$. Again, using (20), we obtain that

$$
\begin{aligned}
C_{1}(M) & \geq \frac{1}{4 \alpha(n-1)(\alpha(n))^{n-1}}\left(\frac{\alpha(n)}{\int_{0}^{d} r^{n-1} d r}\right)^{n+1} \\
& =\frac{(\alpha(n))^{2}}{4 \alpha(n-1)} \cdot \frac{n^{n+1}}{d^{n(n+1)}} .
\end{aligned}
$$

10. It remains to estimate the upper bound of diameter of $M$.

From S. Y. Cheng [1], we know that if the Ricci curvature of compact Riemannian manifold $M^{n}$ is nonnegative, then

$$
\lambda_{1} d^{2} \leq 2 n(n+4)
$$

where $d$ is the diameter of $M, \lambda_{1}$ is the first nonzero eigenvalue of Laplacian for the functions of $M$. Now we must estimate the positive lower bound of $\lambda_{1}(M)$. S. T. Yau [8] has conjectured that $\lambda_{1}(M)=n$ for the compact minimal hypersurface $M^{n}$ in $S^{n+1}(1)$, but so far it has not been proved. Recently, H. I. Choi and A. N. Wang [2] proved that

$$
\lambda_{1}(M) \geq n / 2 .
$$

Hence we get the upper bound of $d$,

$$
d \leq 2 \sqrt{n+4} .
$$

Therefore, from (22), we obtain the positive lower bound of $C_{1}(M)$ :

$$
C_{1}(M) \geq \frac{(\alpha(n))^{2}}{4 \alpha(n-1)} \cdot \frac{n^{n+1}}{(2 \sqrt{n+4})^{n(n+1)}} .
$$


11. When $n \geq 3$, from (18), (26), we can take

$$
\begin{aligned}
& A(n)=\min \left(\frac{n-2}{2(n-1)}\right)^{2} \cdot 2^{-(n+2) / n} \\
& \times\left(\frac{(\alpha(n))^{2}}{4 \alpha(n-1)} \cdot \frac{n^{n+1}}{(2 \sqrt{n+4})^{n(n+1)}}\right)^{2 / n}, \\
&\left.n \cdot 2^{-((n+4) / n+E(n))}(\alpha(n))^{2 / n}\right) .
\end{aligned}
$$

12. When $n=2$, from (19), we know that it is necessary to estimate the upper bound of the volume $\operatorname{vol} M$ of $M^{2}$.

Since $M$ is a Riemann surface with genus $g$, according to Yang and Yau [7], we know that vol $M$ of compact Riemann surface $M$ with genus $g$ will satisfy

$$
\lambda_{1}(M) \cdot \operatorname{vol} M \leq 8 \pi(g+1) .
$$

Because $\lambda_{1}(M) \geq 1$, from H. I. Choi and A. N. Wang [2], we have

$$
\operatorname{vol} M \leq 8 \pi(g+1) \text {. }
$$

Therefore from (19), (26), (28), we can take

$$
\begin{aligned}
A & =\min \left(\frac{1}{4} \cdot \frac{(\alpha(2))^{2}}{4 \alpha(1)} \cdot \frac{2^{3}}{(2 \sqrt{6})^{6}} \cdot \frac{1}{\sqrt{8 \pi(g+1)}},(\alpha(2))^{1 / 2}\right) \\
& =\frac{1}{6912 \sqrt{2 \pi(g+1)}} \cdot \text { Q.E.D. }
\end{aligned}
$$

Remarks. 1. Constants $A(n), A$ in Theorems 1 and 2 are not the best constants.

2. In the higher codimensional case we can reach the step similar to expression (23). But because of the lack of the lower bound estimate of $\lambda_{1}(M)$ (something like Choi and Wang's estimate), we cannot get the final conclusion. If the positive lower bound of $\lambda_{1}(M)$ for the higher dimensional case can be estimated, we can then get the similar theorem about global pinching.

3. In this paper we put the assumption of Ricci $\geq 0$ for $M$. It is equivalent to the condition that $\mu<n-1$, where $\mu$ is the biggest eigenvalue of $h_{i j}^{(2)}$, and $h_{i j}^{(2)}=h_{i k} h_{k j}$ is the square tensor of the second fundamental form of $M^{n}$. The object of adding this condition is to obtain the positive lower bound of the isoperimetric constant.

A very interesting and unsolved problem is whether there is a positive lower bound of the isoperimetric constant for any compact minimal hypersurfaces of sphere and how to estimate this positive lower bound.

4. We can take the similar discussion for the compact complex submanifolds $M^{n}$ in $\mathbf{C} P^{n+p}(1)$. We can reach the step similar to expression (23). If we can get the lower bound estimate of $\lambda_{1}(M)$ (something like Choi and Wang's estimate), then we can obtain the corresponding global pinching theorem in the complex case. 


\section{REFERENCES}

1. S. Y. Cheng, Eigenvalue comparison theorem and its geometric applications, Math. Z. 143 (1975), 289-297.

2. H. I. Choi and A. N. Wang, A first eigenvalue estimate for minimal hypersurfaces, J. Differential Geometry 18 (1983), 559-562.

3. C. B. Croke, Some isoperimetric inequalities and eigenvalue estimates, Ann. Sci. École Norm. Sup. (4) 13 (1980), 419-435.

4. D. Hoffman and J. Spruck, Sobolev and isoperimetric inequalities for Riemannian submanifolds, Comm. Pure Appl. Math. 27 (1975), 715-725.

5. P. Li, On the Sobolev constant and the p-spectrum of a compact Riemannian manifold, Ann. Sci. École Norm. Sup. (4) 13 (1980), 451-467.

6. J. Simons, Minimal varieties in Riemannian manifolds, Ann. of Math. 88 (1968), 62-105.

7. P. Yang and S. T. Yau, Eigenvalue of the Laplacian of compact Riemann surfaces and minimal submanifolds, Ann. Scuola Norm. Sup. Pisa 7 (1980), 55-63.

8. S. T. Yau, Seminar on differential geometry, Ann. of Math. Studies, No. 102, Princeton Univ. Press, Princeton, N.J., 1982.

9. __ Submanifolds with constant mean curvature. II, Amer. J. Math. 97 (1975), 76-100.

Department of Mathematics, Fudan University, Shanghai, People's Republic of China 